\title{
CONSTRAINTS ON SCALAR DARK MATTER FROM DIRECT EXPERIMENTAL SEARCHES*
}

\author{
XIAO-GANG HE ${ }^{1}$, TONG LI ${ }^{2,3}$, XUE-QIAN LI ${ }^{2}$, JUSAK TANDEAN ${ }^{1}$, HO-CHIN TSAI $^{1}$ \\ ${ }^{1}$ Department of Physics, Center for Theoretical Sciences, and LeCosPA Center, \\ National Taiwan University, Taipei 10617, Taiwan \\ ${ }^{2}$ Department of Physics, Nankai University, Tianjin 300071, China \\ ${ }^{3}$ Center for High Energy Physics, Peking University, Beijing 100871, China
}

\begin{abstract}
The standard model (SM) plus a real gauge-singlet scalar field dubbed darkon (SM+D) is the simplest model possessing a weakly interacting massive particle (WIMP) dark-matter candidate. The upper limits for the WIMP-nucleon elastic cross-section as a function of WIMP mass from the recent XENON10 and CDMS II experiments rule out darkon mass ranges from 10 to $(50,70,75) \mathrm{GeV}$ for Higgs-boson masses of $(120,200,350) \mathrm{GeV}$, respectively. This may exclude the possibility of the darkon providing an explanation for the gamma-ray excess observed in the EGRET data. We show that by extending the $\mathrm{SM}+\mathrm{D}$ to a two-Higgs-doublet model plus a darkon the experimental constraints on the WIMP-nucleon interactions can be circumvented due to suppression occurring at some values of the product $\tan \alpha \tan \beta$, with $\alpha$ being the neutral-Higgs mixing angle and $\tan \beta$ the ratio of vacuum expectation values of the Higgs doublets. We also comment on the implication of the darkon model for Higgs searches at the LHC.
\end{abstract}

Keywords: Darkon; dark matter; Higgs; LHC; THDM II.

PACS Nos.: 95.35.+d, 95.30.Cq, 14.80.Bn, 14.80.Cp

\section{Introduction}

The simplest model which has a weakly interacting massive particle (WIMP) candidate is the $\mathrm{SM}+\mathrm{D}$, which extends the $\mathrm{SM}$ by the addition of a real gauge-singlet scalar field $D$ which we call darkon. Darkon as dark matter (DM) was first considered by Silveira and Zee ${ }^{1}$ and further explored later by other groups. ${ }^{2,3,4,5}$

Here we report the results of a recent study ${ }^{6}$ on the $\mathrm{SM}+\mathrm{D}$ and its extension two-Higgs-doublet model type-II plus a darkon (THDM II+D) subjected to cold DM relic and direct DM search experiments. To be DM the darkon should reproduce the correct cold DM relic. Also at the same time the darkon should be able to satisfy constraints from recent DM direct search experiments XENON10 ${ }^{7}$ and $\mathrm{CDMS} \mathrm{II}^{8}$ which have set the strictest upper limits to date for the WIMP-nucleon spin-independent elastic scattering cross-section as a function of WIMP mass.

*Presented by H.C. Tsai. 
We find that the $\mathrm{SM}+\mathrm{D}$ with darkon mass values within the ranges of $10 \mathrm{GeV}$ to $(50,70,75) \mathrm{GeV}$ for Higgs masses of $(120,200,350) \mathrm{GeV}$, respectively, is ruled out

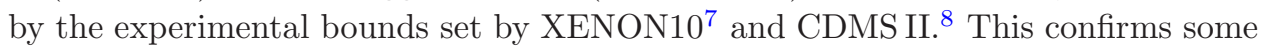
of the results obtained in Ref. 5. But it would imply that the darkon model is not likely to offer an explanation for the EGRET excess, where a WIMP candidate with a mass in the range from 50 to $70 \mathrm{GeV}$ can well explain the gamma-ray excess observed in the EGRET data. ${ }^{9}$ We also find that a darkon in the THDM II+D can evade the recent experimental restrictions to keep the mass range of interest viable. In addition we also explore some of the implications of the darkon model for Higgs searches at the LHC.

\section{Standard Model with Darkon}

\subsection{Brief description of the model}

To play the role of DM, we require a darkon to be a real gauge singlet and its Lagrangian to satisfy a discrete $Z_{2}$ symmetry $D \rightarrow-D$. Also requiring the darkon interactions to be renormalizable implies that $D$ can only couple to the Higgs doublet field $H$. Beside the kinetic energy term $\frac{1}{2} \partial^{\mu} D \partial_{\mu} D$, the general form of the other terms in the darkon Lagrangian is ${ }^{1,2}$

$$
\mathcal{L}_{D}=-\frac{\lambda_{D}}{4} D^{4}-\frac{m_{0}^{2}}{2} D^{2}-\lambda D^{2} H^{\dagger} H
$$

where $\lambda_{D}, m_{0}$, and $\lambda$ are free parameters. The parameters in the potential should be chosen such that $D$ does not develop a vacuum expectation value (vev) and the $Z_{2}$ symmetry is not broken, which will ensure that the darkon does not mix with the Higgs field to avoid its possible fast decay. Then Eq. (1) can be rewritten to describe the interaction of the physical Higgs boson $h$ with the darkon as

$$
\mathcal{L}_{D}=-\frac{\lambda_{D}}{4} D^{4}-\frac{\left(m_{0}^{2}+\lambda v^{2}\right)}{2} D^{2}-\frac{\lambda}{2} D^{2} h^{2}-\lambda v D^{2} h,
$$

where $v=246 \mathrm{GeV}$ is the vev of $H$, the second term contains the darkon mass $m_{D}=\left(m_{0}^{2}+\lambda v^{2}\right)^{1 / 2}$. And the last term, $-\lambda v D^{2} h$, at leading order can introduce the annihilation of a darkon pair into SM particles through Higgs exchange, ${ }^{1,2}$ namely $D D \rightarrow h^{*} \rightarrow X$, where $X$ indicates SM particles to determine the relic density of the darkon.

Since the darkon is cold DM, its speed is nonrelativistic, and so a darkon pair has an invariant mass $\sqrt{s} \simeq 2 m_{D}$. With the $\mathrm{SM}+\mathrm{D}$ Lagrangian determined, the $h$-mediated annihilation cross-section of a darkon pair into SM particles can be obtained $\mathrm{as}^{2}$

$$
\sigma_{\text {ann }} v_{\text {rel }}=\frac{8 \lambda^{2} v^{2}}{\left(4 m_{D}^{2}-m_{h}^{2}\right)^{2}+\Gamma_{h}^{2} m_{h}^{2}} \frac{\sum_{i} \Gamma\left(\tilde{h} \rightarrow X_{i}\right)}{2 m_{D}},
$$

where $v_{\text {rel }}=2\left|\boldsymbol{p}_{D}^{\mathrm{cm}}\right| / m_{D}$ is the relative speed of the $D D$ pair in their center-ofmass $(\mathrm{cm})$ frame, $\tilde{h}$ is a virtual Higgs boson with an invariant mass $\sqrt{s}=2 m_{D}$, 
and $\tilde{h} \rightarrow X_{i}$ is any possible decay mode of $\tilde{h}$. For a given model, $\Sigma_{i} \Gamma\left(\tilde{h} \rightarrow X_{i}\right)$ is obtained by calculating the $h$ width and then setting $m_{h}$ equal to $2 m_{D}$.

To a good approximation the thermal average annihilation cross-section $\left\langle\sigma_{\text {ann }} v_{\text {rel }}\right\rangle$ is bonded to the cold DM relic by thermal dynamic relations of Universe in the standard big-bang cosmology ${ }^{10}$

$$
\Omega_{D} h^{2} \simeq \frac{1.07 \times 10^{9} x_{f}}{\sqrt{g_{*}} m_{\mathrm{Pl}}\left\langle\sigma_{\mathrm{ann}} v_{\mathrm{rel}}\right\rangle \mathrm{GeV}}, \quad x_{f} \simeq \ln \frac{0.038 m_{\mathrm{Pl}} m_{D}\left\langle\sigma_{\mathrm{ann}} v_{\mathrm{rel}}\right\rangle}{\sqrt{g_{*} x_{f}}}
$$

$m_{\mathrm{Pl}}=1.22 \times 10^{19} \mathrm{GeV}$ is the Planck mass, $x_{f}=m_{D} / T_{f}$ with $T_{f}$ being the freezing temperature, $g_{*}$ is the number of relativistic degrees of freedom with masses less than $T_{f}$. The current value of the relic density is $\Omega_{D} h^{2}=0.105 \pm 0.008$ quoted from Particle Data Group ${ }^{11}$ here $h$ is the Hubble constant in units of $100 \mathrm{~km} /(\mathrm{s} \cdot \mathrm{Mpc})$.

Using the relic density number and Eq. (4) we can find the allowed range of the darkon-Higgs coupling $\lambda$ as a function of $m_{D}$. Numerically we adopt 90\% C.L. range of $0.092 \leq \Omega_{D} h^{2} \leq 0.118 .{ }^{11}$ In Fig. 1(a) we display the allowed $\lambda$ values for $10 \mathrm{GeV} \leq m_{D} \leq 100 \mathrm{GeV}$ and representative values of the Higgs-boson mass.
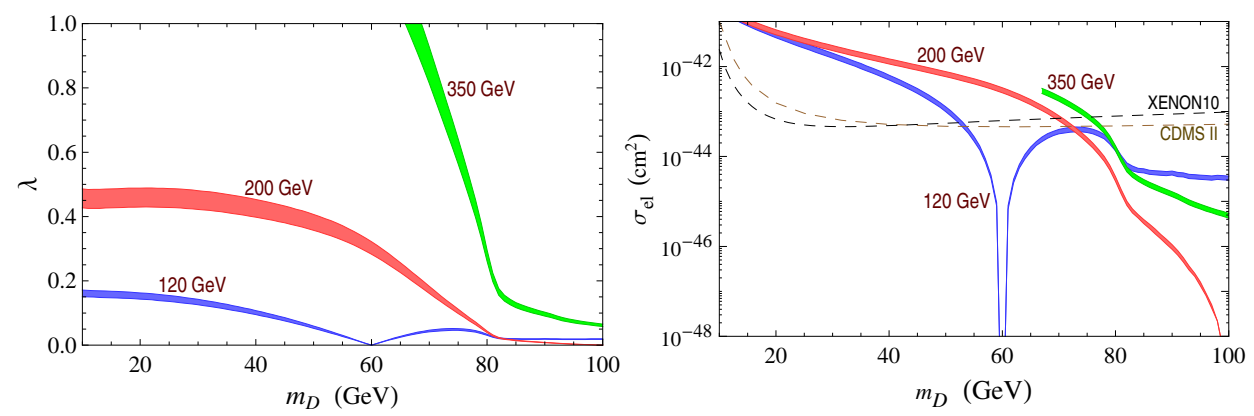

Fig. 1. (Color online) (a) Left plot: Darkon-Higgs coupling $\lambda$ in the SM+D as a function of darkon mass $m_{D}$ for Higgs mass values $m_{h}=120,200,350 \mathrm{GeV}$. (b) Right plot: Darkon-nucleon elastic cross-section $\sigma_{\text {el }}$ in the $\mathrm{SM}+\mathrm{D}$ as a function of darkon mass $m_{D}$ for Higgs mass values $m_{h}=120,200,350 \mathrm{GeV}$, compared to $90 \%$ C.L. upper limits from XENON10 (black dashed-curve) and CDMS II (brown dashed-curve).

\subsection{Darkon-nucleon elastic cross-section in $S M+D$}

Direct detection of DM on the earth is through the measurement of nuclear recoil when a darkon hits a nucleon. This occurs via the $t$-channel elastic scattering $D N \rightarrow$ $D N$ mediated by a Higgs boson $\mathcal{H}$ whose amplitude is given by

$$
\mathcal{M}_{\mathrm{el}} \simeq \frac{2 \lambda g_{N N \mathcal{H}} v}{m_{\mathcal{H}}^{2}} \bar{N} N
$$


under the squared transfer momentum $t \ll m_{\mathcal{H}}^{2}$, where $g_{N N \mathcal{H}}, N=p$ or $n$ is the Higgs-nucleon coupling. This leads to the cross section

$$
\sigma_{\mathrm{el}} \simeq \frac{\lambda^{2} g_{N N \mathcal{H}}^{2} v^{2} m_{N}^{2}}{\pi\left(m_{D}+m_{N}\right)^{2} m_{\mathcal{H}}^{4}}
$$

in the approximation of $\left(p_{D}+p_{N}\right)^{2} \simeq\left(m_{D}+m_{N}\right)^{2}$.

Since the energy transferred in this elastic scattering is very small, of order $100 \mathrm{keV}$, the effective Higgs-nucleon coupling needed can be obtained via a chiralLagrangian approach. The effective Higgs-nucleon interaction Lagrangian is written as

$$
\mathcal{L}_{N N \mathcal{H}}=-g_{N N \mathcal{H}} \bar{N} N \mathcal{H}
$$

which depends on the underlying Yukawa interactions of the Higgs and quarks as

$$
\mathcal{L}_{q q \mathcal{H}}=-\sum_{q} \frac{k_{q}}{v} m_{q} \bar{q} q \mathcal{H}
$$

where in $\mathrm{SM} k_{q}=1$ for all q's. For the $\mathrm{SM}+\mathrm{D}$ and THDM II $+\mathrm{D}$, we can evaluate the matrix element ${ }^{12,13}$ below

$$
g_{N N \mathcal{H}} \bar{N} N=\left\langle N\left|\frac{k_{u}}{v}\left(m_{u} \bar{u} u+m_{c} \bar{c} c+m_{t} \bar{t} t\right)+\frac{k_{d}}{v}\left(m_{d} \bar{d} d+m_{s} \bar{s} s+m_{b} \bar{b} b\right)\right| N\right\rangle
$$

to obtain Higgs-nucleon coupling 6

$$
g_{N N \mathcal{H}}=\left(k_{u}-k_{d}\right) \frac{\sigma_{\pi N}}{2 v}+k_{d} \frac{m_{N}}{v}+\frac{4 k_{u}-25 k_{d}}{27} \frac{m_{B}}{v},
$$

where $\sigma_{\pi N}$ is the so-called pion-nucleon sigma term, $m_{N}$ the nucleon mass, and $m_{B}$ the baryon mass in the chiral limit. Empirically, $\sigma_{\pi_{N}}$ is not very precisely determined and hence will be a source of uncertainty in our calculation. For definiteness we adopt $\sigma_{\pi N}=45 \mathrm{MeV} \cdot{ }^{14}$ It follows that

$$
g_{N N \mathcal{H}} \simeq\left(1.217 k_{d}+0.493 k_{u}\right) \times 10^{-3}
$$

In the $\mathrm{SM}+\mathrm{D}$, we have $k_{u}=k_{d}=1$ and so $g_{N N h}^{\mathrm{SM}} \simeq 1.71 \times 10^{-3}$ which is comparable to the values found in the literature. ${ }^{2,15}$ With $g_{N N h}^{\mathrm{SM}}$ known and $\lambda$ also known as a function of $m_{D}$ from the relic density constraint, one can predict $\sigma_{\mathrm{el}}$. Thus we plot in Fig. 1(b) $\sigma_{\text {el }}$ versus $m_{D}$ of the $\mathrm{SM}+\mathrm{D}$ for representative values of the Higgs-boson mass. In Fig. 1(b), we also plot 90\% C.L. upper limits on the WIMPnucleon spin-independent elastic cross-section set by the XENON10 and CDMS II experiments. $^{7,8}$ One can easily see that the darkon mass ranges $10 \mathrm{GeV} \leq m_{D} \leq$ $(50,70,75) \mathrm{GeV}$ are ruled out for Higgs masses of $(120,200,350) \mathrm{GeV}$. 


\section{Two-Higgs-Doublet Model with Darkon}

\subsection{Brief description of the model}

Depending on how the two Higgs doublets $H_{1,2}$ couple to the fermions in the SM there are different types of the THDM. It turns out the type II of the THDM, in which the up type fermions get mass from only one of the Higgs doublets and the down type fermions from the other doublet, has the desired features for our purposes. Therefore we will consider only the THDM II with the darkon field added $($ THDM II+D). In this case the Yukawa interactions of the Higgs fields are described by $^{16}$

$$
\mathcal{L}_{\mathrm{Y}}=-\bar{Q}_{L} \lambda_{2}^{u} \tilde{H}_{2} \mathcal{U}_{R}-\bar{Q}_{L} \lambda_{1}^{d} H_{1} \mathcal{D}_{R}-\bar{L}_{L} \lambda_{1}^{l} H_{1} E_{R}+\text { H.c. },
$$

where $Q, \mathcal{U}, \mathcal{D}, L$, and $E$ represent the usual quark and lepton fields and $\lambda^{u, d, l}$ contain the Yukawa couplings. To effect the separate couplings of $H_{1}$ and $H_{2}$ to the down and up sectors, respectively, it is necessary to introduce a discrete $Z_{2}^{\prime}$ symmetry, under which $H_{2} \rightarrow-H_{2}$ and $\mathcal{U}_{R} \rightarrow-\mathcal{U}_{R}$, the other fields being unaltered. In terms of their components, the Higgs doublets are

$$
H_{k}=\frac{1}{\sqrt{2}}\left(\begin{array}{c}
\sqrt{2} h_{k}^{+} \\
v_{k}+h_{k}+i I_{k}
\end{array}\right),
$$

where $k=1,2$ and $v_{k}$ is the vev of $H_{k}$. Here $h_{k}^{+}$and $I_{k}$ are related to the physical Higgs bosons $H^{+}$and $A$ and the would-be Goldstone bosons $w$ and $z$ by

$$
\left(\begin{array}{l}
h_{1}^{+} \\
h_{2}^{+}
\end{array}\right)=\left(\begin{array}{rr}
\cos \beta & -\sin \beta \\
\sin \beta & \cos \beta
\end{array}\right)\left(\begin{array}{l}
w^{+} \\
H^{+}
\end{array}\right),\left(\begin{array}{l}
I_{1} \\
I_{2}
\end{array}\right)=\left(\begin{array}{rr}
\cos \beta & -\sin \beta \\
\sin \beta & \cos \beta
\end{array}\right)\left(\begin{array}{l}
z \\
A
\end{array}\right)
$$

with $\tan \beta=v_{2} / v_{1}$, whereas $h_{k}$ in terms of mass eigenstates $H$ and $h$ as

$$
\left(\begin{array}{l}
h_{1} \\
h_{2}
\end{array}\right)=\left(\begin{array}{rr}
\cos \alpha & -\sin \alpha \\
\sin \alpha & \cos \alpha
\end{array}\right)\left(\begin{array}{l}
H \\
h
\end{array}\right)
$$

Hence the angle $\alpha$ indicates the mixing of the two $C P$-even Higgs bosons.

The Yukawa couplings of $h$ and $H$ can be obtained from Eq. (12)

$$
\begin{aligned}
\mathcal{L}_{f f \mathcal{H}}= & -\overline{\mathcal{U}}_{L} M^{u} \mathcal{U}_{R}\left(\frac{\cos \alpha}{\sin \beta} \frac{h}{v}+\frac{\sin \alpha}{\sin \beta} \frac{H}{v}\right)-\overline{\mathcal{D}}_{L} M^{d} \mathcal{D}_{R}\left(-\frac{\sin \alpha}{\cos \beta} \frac{h}{v}+\frac{\cos \alpha}{\cos \beta} \frac{H}{v}\right) \\
& -\bar{E}_{L} M^{l} E_{R}\left(-\frac{\sin \alpha}{\cos \beta} \frac{h}{v}+\frac{\cos \alpha}{\cos \beta} \frac{H}{v}\right)+\text { H.c. . }
\end{aligned}
$$

The weak bosons $V=W^{ \pm}, Z$ couplings of $h$ and $H$ can be obtained from the kinetic sector of the $\mathrm{THDM}^{16}$

$$
\mathcal{L}_{V V \mathcal{H}}=\left(\frac{2 m_{W}^{2}}{v} W^{+\mu} W_{\mu}^{-}+\frac{m_{Z}^{2}}{v} Z^{\mu} Z_{\mu}\right)[h \sin (\beta-\alpha)+H \cos (\beta-\alpha)] .
$$


In analogy to Eq. (1) in the SM+D case, in the THDM II+D we have the renormalizable darkon Lagrangian

$$
\mathcal{L}_{D}=-\frac{\lambda_{D}}{4} D^{4}-\frac{m_{0}^{2}}{2} D^{2}-\left(\lambda_{1} H_{1}^{\dagger} H_{1}+\lambda_{2} H_{2}^{\dagger} H_{2}\right) D^{2}
$$

which also respects to unbroken $Z_{2}$ and $Z_{2}^{\prime}$ symmetries mentioned above. After electroweak symmetry breaking we obtain

$$
\begin{aligned}
\mathcal{L}_{D D h} & =-\left(-\lambda_{1} \sin \alpha \cos \beta+\lambda_{2} \cos \alpha \sin \beta\right) v D^{2} h=-\lambda_{h} v D^{2} h, \\
\mathcal{L}_{D D H} & =-\left(\lambda_{1} \cos \alpha \cos \beta+\lambda_{2} \sin \alpha \sin \beta\right) v D^{2} H=-\lambda_{H} v D^{2} H,
\end{aligned}
$$

but no $D D A$ term. The effective couplings $\lambda_{h, H}$ are free parameters because $\lambda_{1}$, and $\lambda_{2}$ are also free parameters.

\subsection{Darkon-nucleon elastic cross-section in $T H D M I I+D$}

Using the formulas given above, one can express the cross-section of the darkonnucleon elastic scattering in the THDM II+D as

$$
\sigma_{\mathrm{el}} \simeq \frac{m_{N}^{2} v^{2}}{\pi\left(m_{D}+m_{N}\right)^{2}}\left(\frac{\lambda_{h} g_{N N h}^{\mathrm{THDM}}}{m_{h}^{2}}+\frac{\lambda_{H} g_{N N H}^{\mathrm{THDM}}}{m_{H}^{2}}\right)^{2},
$$

where, from Eqs. (10) and (11), the nucleon coupling to $\mathcal{H}=h$ or $H$ is

$$
\begin{aligned}
g_{N N \mathcal{H}}^{\mathrm{THDM}} & =\left(k_{u}^{\mathcal{H}}-k_{d}^{\mathcal{H}}\right) \frac{\sigma_{\pi N}}{2 v}+k_{d}^{\mathcal{H}} \frac{m_{N}}{v}+\frac{4 k_{u}^{\mathcal{H}}-25 k_{d}^{\mathcal{H}}}{27} \frac{m_{B}}{v} \\
& \simeq\left(1.217 k_{d}^{\mathcal{H}}+0.493 k_{u}^{\mathcal{H}}\right) \times 10^{-3} .
\end{aligned}
$$

The parameters $k_{q}^{\mathcal{H}}$ can be read off from Eq. (16). For simplicity we require $\lambda_{H}$ to be very small or $m_{H}$ very large to ignore contributions from $H$. So from Eq. (21) we see the Higgs-nucleon coupling $g_{N N h}^{\mathrm{THDM}}$ can vanish since the two relevant parameters $k_{u, d}^{h}$ are free in the THDM II+D and the cancelation condition is

$$
\frac{k_{d}^{h}}{k_{u}^{h}}=-\tan \alpha \tan \beta \simeq-0.405 .
$$

For illustration, we display in Fig. 2 the $\lambda_{h}$ values allowed by the relic density constraint for two cases satisfying $\tan \alpha \tan \beta=0.45$ which can lead to the correct DM relic density. The corresponding darkon-nucleon elastic cross-section $\sigma_{\mathrm{el}}$ is shown in Fig. 3. Also shown are the upper limits from XENON10 and CDMSII, along with the expected sensitivities of a number of future experiments. ${ }^{17}$

\section{Discussions and Conclusions}

The existence of the darkon can give rise to huge enhancement of the Higgs width via the additional process $h \rightarrow D D$ and hence affect Higgs searches at the LHC. For a Higgs boson with a large invisible branching fraction $(>60 \%)$ and a mass within the range $120 \mathrm{GeV} \lesssim m_{h} \lesssim 300 \mathrm{GeV}$, direct searches at CMS through the 

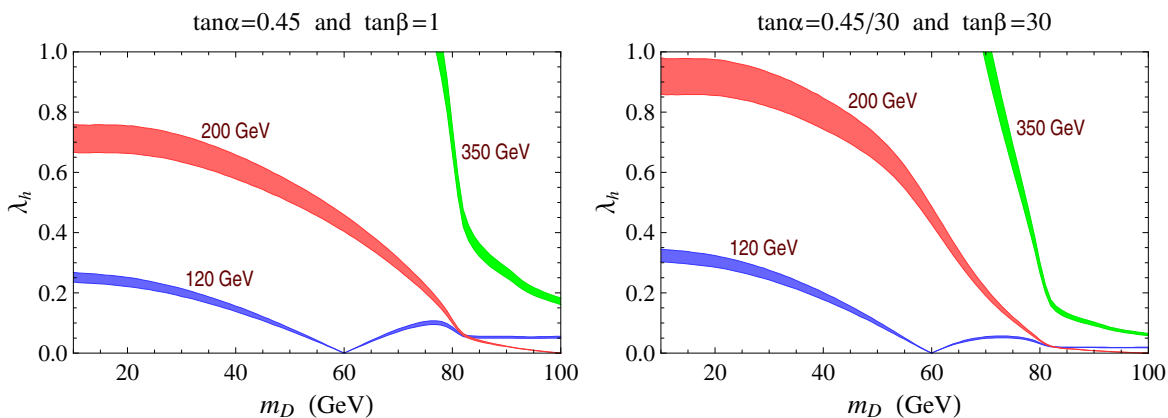

Fig. 2. (Color online) Darkon-Higgs coupling $\lambda_{h}$ in the THDM II+D as a function of darkon mass $m_{D}$ for Higgs mass values $m_{h}=120,200,350 \mathrm{GeV}$ for two cases with different $\tan \alpha$ and $\tan \beta$ values satisfying $\tan \alpha \tan \beta=0.45$. Only regions corresponding to $\lambda_{h}<1$ have been plotted.
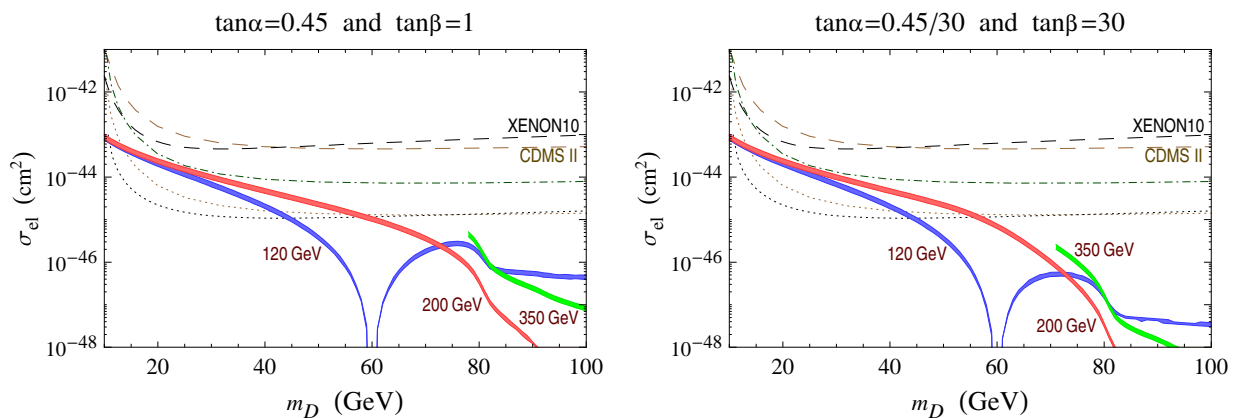

Fig. 3. (Color online) Darkon-nucleon elastic cross-section $\sigma_{\text {el }}$ in the THDM II+D for the two cases in Fig. 2, compared to the 90\% C.L. upper limits from XENON10 (black dashed curve) and CDMS II (brown dashed curve), as well as projected sensitivities of SuperCDMS at Soudan (green dot-dashed curve), SuperCDMS at Snolab (brown dotted curve), and XENON100 (black dotted curve).

usual SM modes may be unfeasible with $30 \mathrm{fb}^{-1}$ of integrated luminosity. ${ }^{5}$ However, such a Higgs boson can be observed at ATLAS with the same integrated luminosity, via weak-boson fusion or $Z$-Higgstrahlung, by looking for missing energy from the decay. ${ }^{5,18}$ Considering one of our THDM II + D examples, with $\tan \alpha=0.015$ and $\tan \beta=30$, we find that $h$ has SM-like couplings to the weak bosons and invisible branching ratios higher than 0.6 in much of the $m_{D}$ range, which serves to illustrate the testability of the darkon model at the LHC. Moreover, in the SM+D, if $m_{h}>$ $2 m_{Z}$, say $m_{h}=300 \mathrm{GeV}$, the total Higgs width can be measured with a precision of up to $10 \%$ at ATLAS with $300 \mathrm{fb}^{-1}$ integrated luminosity, ${ }^{19}$ and then the darkon contribution can be inferred after a comparison with the SM prediction for the width.

In conclusion, we have shown that the $\mathrm{SM}+\mathrm{D}$ with darkon mass values within the ranges of $10 \mathrm{GeV}$ to $(50,70,75) \mathrm{GeV}$ for Higgs masses of $(120,200,350) \mathrm{GeV}$, 
respectively, is ruled out by the experimental bounds set by XENON10 and CDMS II. We have also shown that in the THDM II+D the experimental constraints can be evaded due to suppression of the darkon-nucleon elastic cross-section at some values of $\tan \alpha \tan \beta$. The model can be further constrained by future DM search experiments. Finally the substantial increase of the invisible decay width of the Higgs boson would lead to a sizable reduction of its branching fraction to SM particles. Although this could significantly affect Higgs searches at the LHC, we expect that it will still be able to probe the darkon model.

\section{Acknowledgments}

This work was partially supported by NSC, NCTS, and NNSF.

\section{References}

1. V. Silveira and A. Zee, Phys. Lett. B 161, 136 (1985).

2. C. P. Burgess, M. Pospelov and T. ter Veldhuis, Nucl. Phys. B 619, 709 (2001) [arXiv:hep-ph/0011335].

3. J. McDonald, Phys. Rev. D 50, 3637 (1994) [arXiv:hep-ph/0702143]; M. C. Bento, O. Bertolami, R. Rosenfeld and L. Teodoro, ibid. 62, 041302 (2000) [arXiv:astroph/0003350]; D. E. Holz and A. Zee, Phys. Lett. B 517, 239 (2001) [arXiv:hepph/0105284]. M. C. Bento, O. Bertolami and R. Rosenfeld, ibid. 518, 276 (2001) [arXiv:hep-ph/0103340]; J. McDonald, Phys. Rev. Lett. 88, 091304 (2002) [arXiv:hepph/0106249]; C. Bird, P. Jackson, R. Kowalewski and M. Pospelov, ibid. 93, 201803 (2004) [arXiv:hep-ph/0401195]; H. Davoudiasl, R. Kitano, T. Li and H. Murayama, Phys. Lett. B 609, 117 (2005) [arXiv:hep-ph/0405097]. G. Cynolter, E. Lendvai and G. Pocsik, Acta Phys. Polon. B 36, 827 (2005) [arXiv:hep-ph/0410102]; C. Bird, R. Kowalewski and M. Pospelov, Mod. Phys. Lett. A 21, 457 (2006) [arXiv:hep-ph/0601090]; S. H. Zhu, arXiv:hep-ph/0601224; S. Andreas, T. Hambye and M. H. G. Tytgat, JCAP 0810, 034 (2008) [arXiv:0808.0255 [hep-ph]]. C. E. Yaguna, arXiv:0810.4267 [hep-ph].

4. X. G. He, T. Li, X.Q. Li and H.C. Tsai, Mod. Phys. Lett. A 22, 2121 (2007) [arXiv:hep$\mathrm{ph} / 0701156]$.

5. V. Barger, P. Langacker, M. McCaskey, M.J. Ramsey-Musolf and G. Shaughnessy, Phys. Rev. D 77, 035005 (2008) [arXiv:0706.4311 [hep-ph]].

6. X. G. He, T. Li, X. Q. Li, J. Tandean and H. C. Tsai, arXiv:0811.0658 [hep-ph], to appear in Phys. Rev. D.

7. J. Angle et al. [XENON Collaboration], Phys. Rev. Lett. 100, 021303 (2008) [arXiv:0706.0039 [astro-ph]].

8. Z. Ahmed et al. [CDMS Collaboration], arXiv:0802.3530 [astro-ph].

9. W. de Boer, C. Sander, V. Zhukov, A.V. Gladyshev and D.I. Kazakov, Astron. Astrophys. 444, 51 (2005) [arXiv:astro-ph/0508617].

10. E. W. Kolb and M. Turner, The Early Universe (Westview Press, Boulder, 1990).

11. C. Amsler et al. [Particle Data Group], Phys. Lett. B 667, 1 (2008).

12. J. Gasser and H. Leutwyler, Annals Phys. 158, 142 (1984); J. Bijnens, H. Sonoda and M. B. Wise, Nucl. Phys. B 261, 185 (1985); E. Jenkins and A. V. Manohar, in Effective Field Theories of the Standard Model, edited by U.-G. Meissner (World Scientific, Singapore, 1992). 
13. See, e.g., J. F. Donoghue, E. Golowich and B.R. Holstein, Dynamics of the Standard Model (Cambridge University Press, Cambridge, 1992).

14. J. Gasser, H. Leutwyler and M. E. Sainio, Phys. Lett. B 253, 252 (1991).

15. T. P. Cheng, Phys. Rev. D 38, 2869 (1988); H. Y. Cheng, Phys. Lett. B 219, 347 (1989).

16. For review, see J. F. Gunion, H. E. Haber, G. L. Kane and S. Dawson, The Higgs Hunter's Guide (Westview Press, Colorado, 2000).

17. R. Gaitskell, V. Mandic and J. Filippini, http://dmtools.berkeley.edu/limitplots.

18. H. Davoudiasl, T. Han and H.E. Logan, Phys. Rev. D 71, 115007 (2005) [arXiv:hep$\mathrm{ph} / 0412269]$.

19. ATLAS Collaboration, Report No. CERN/LHCC 99-15. 